\title{
Variations de résistance des sols compactés avec la pression interstitielle négative
}

\author{
Strength of compacted soils in relation \\ to the negative pore pressure
}

\section{J.-M. FLEUREAU}

Chef de travaux

S. KHEIRBEK-SAOUD

Chercheur

Ecole centrale de Paris, Laboratoire de Mécanique (Sols, Structures et Matériaux), CNRS URA 850 \& GRECO 90 Géomatériaux

Rev. Franç. Géotech. n 59, pp. 57-64 (avril 1992)

\section{Résumé}

L'étude réalisée sur trois matériaux argileux dont les limites de liquidité sont comprises entre 40 et $82 \%$ a montré qu'à contrainte totale constante, l'indice CBR pouvait s'exprimer comme une fonction puissance de la pression interstitielle négative (succion). Si l'on prend en compte les courbes d'humidification, cette relation permet de prévoir l'évolution de la résistance du sol compacté en cas de pluies ou d'inondation.

En outre, une étude statistique, effectuée sur de nombreux matériaux de chaussée a conduit à une classification des matériaux convenant bien à la réalisation des routes (matériaux contenant moins de $15 \%$ de fins $<80 \mu \mathrm{m}$ ), des matériaux mal adaptés (plus de $50 \%$ de fins) et des matériaux intermédiaires nécessitant une étude plus approfondie.

\footnotetext{
Abstract

The study of three materials, whose liquid limits ranged from 40 to $82 \%$ showed that, for constant total stresses, the CBR ratio could be expressed as a power function of the negative pore pressure (suction). Based on this relationship and on the knowledge of the wetting curves of the materials, it is possible to predict the evolution of the strength of a soil in the case of rain or submersion.

Moreover, a statistical study on many different road materials led to a classification of the materials well suited to road construction (materials containing less than $15 \%$ fines $<80 \mu \mathrm{m}$ ), those which were not acceptable (more than $50 \%$ fines) and the intermediate materials, for which a specific study is required.
} 


\section{INTRODUCTION}

La résistance maximale d'un sol non saturé dépend à la fois des contraintes externes qui lui sont appliquées et de la pression interstitielle négative de l'eau (ou «succion»). Deux approches sont utilisées pour aborder le problème :

- soit en considérant les deux paramètres comme indépendants et en déterminant de façon empirique la résistance en fonction de ces deux paramètres (FREDLUND et MORGENSTERN, 1977 ; GULHATI et SATIJA, 1981; ESCARIO et SAEZ, 1986 ; TOLL, 1990) ;

- soit en définissant une contrainte effective fonction des deux paramètres.

Cette approche a été sévèrement critiquée dans la mesure où elle se révèlait apparemment incapable de rendre compte des variations de volume des sols faiblement saturés, et à cause des difficultés de détermination des paramètres de contrainte effective (BLIGHT, 1965: MATYAS et RADHAKRISHNA, 1968). Récemment, BIAREZ et al. (1992) ont montré que la résistance d'un milieu granulaire pouvait s'exprimer en fonction d'une contrainte intergranulaire, définie comme la somme de la contrainte totale externe et d'une contrainte d'origine capillaire dont ils ont proposé une formulation analytique. Dans le cas d'un matériau saturé ou compacté à droite de l'Optimum Proctor Modifié, la contrainte capillaire n'est autre que la pression interstitielle négative et la définition de la contrainte effective de TERZAGHI est applicable (BIAREZ et al., 1991 ; FLEUREAU et INDARTO, 1992).

Lors de la construction d'une route, le sol support est mis en place dans des conditions voisines de l'Optimum Proctor Modifié et sa résistance peut être estimée, par exemple, à partir de l'essai CBR normalisé. L'intérêt de cet essai réside dans sa simplicité et dans l'expérience importante acquise lors de son utilisation pour la réalisation de routes dans le monde entier. La sensibilité du sol à l'eau est déterminée en mesurant la résistance CBR après immersion totale dans l'eau pendant 4 ou 7 jours. Ceci revient à étudier l'évolution de la résistance lorsque la pression interstitielle négative passe d'une valeur initiale de l'ordre de quelques centaines de $\mathrm{kPa}$ à une valeur finale nulle. Or, les mesures faites sous les routes ont montré que, dans les conditions réelles de fonctionnement d'une chaussée, la pression revenait rarement à 0 (SYLVESTRE, 1986 : GEORGE, 1988). Par conséquent, l'essai classique d'immersion conduit généralement à surestimer les épaisseurs de couches.

L'objectif de l'étude présentée dans cet article est de replacer la résistance CBR dans l'ensemble des mesures de lois rhéologiques, d'examiner les variations de cette résistance en fonction de la pression négative sur un chemin d'humidification à partir de l'OPM et enfin, de définir les critères de choix des matériaux routiers selon leur sensibilité à l'eau.

\section{MATÉRIAUX ET MÉTHODES}

\subsection{Identification des matériaux}

Trois matériaux ont été fabriqués pour cette étude en mélangeant dans des proportions variables de l'argile et du sable. Ce sable est composé de $50 \%$ de sable d'Hostun $70 / 270$ (95\% de grains entre 70 et $270 \mu \mathrm{m}$ ) et de $50 \%$ de silice SIKA 40M (100\% de grains inférieurs à $40 \mu \mathrm{m}$ ). Les caractéristiques des mélanges sont indiquées dans le tableau I.

\subsection{Préparation des matériaux et mode opératoire}

Tous les matériaux utilisés ont été préparés par compactage de poudres humides dans les conditions Proctor Modifié normalisées. Les poudres humides sont obtenues en mélangeant l'eau à la poudre sèche, puis en passant le mélange à travers un tamis de $5 \mathrm{~mm}$ d'ouverture, ce qui correspond à des diamètres de " mottes " de l'ordre de quelques millimètres dans l'échantillon final. Le poinçonnement CBR est fait sans surcharge, à une vitesse de $1 \mathrm{~mm} / \mathrm{min}$, d'une part immédiatement après le compactage, d'autre part

Tableau I. - Identification des matériaux étudiés.

\begin{tabular}{|c|c|c|c|}
\hline & Matériau 1 & Matériau 2 & Matériau 3 \\
\hline Composition minéralogique & $100 \%$ kaolinite & $\begin{array}{c}80 \% \text { kaolinite } \\
20 \% \text { sable }\end{array}$ & $\begin{array}{c}80 \% \text { bentonite } \\
20 \% \text { sable }\end{array}$ \\
\hline $\begin{array}{c}\text { Granulométrie : } \\
\%<80 \mu \mathrm{m} \\
\%<2 \mu \mathrm{m} \\
\%<(\mu \mathrm{m}) \\
\mathrm{D}_{60}\left(\mathrm{D}_{10}(\mu \mathrm{m})\right. \\
\mathrm{D}_{60} / \mathrm{D}_{10}\end{array}$ & $\begin{array}{c}100 \\
59 \\
20 \\
\text { env. } 1,5 \\
13\end{array}$ & $\begin{array}{c}93 \\
68 \\
10 \\
\text { env. } 0,6 \\
16\end{array}$ & $\begin{array}{l}93 \\
60 \\
20 \\
\text { env. } 1 \\
20\end{array}$ \\
\hline $\begin{array}{l}\text { Plasticité } \\
\qquad \begin{array}{l}W_{L} \\
W_{p} \\
I_{p}\end{array}\end{array}$ & $\begin{array}{l}40 \\
20 \\
20\end{array}$ & $\begin{array}{l}52 \\
24 \\
28\end{array}$ & $\begin{array}{l}83 \\
30 \\
53\end{array}$ \\
\hline$\gamma_{s} / \gamma_{w}$ & $20^{2,65}$ & $\begin{array}{c}2,65 \\
17.7\end{array}$ & 20 \\
\hline
\end{tabular}


après un mois et demi d'immersion de l'échantillon dans l'eau.

\subsection{Mesure et imposition de la pression négative}

Les chemins d'humidification à partir de l'OPM des différents matériaux ont été déterminés en imposant des pressions négatives données à des échantillons de petite taille par diverses méthodes (surpression d'air, plaques tensiométriques) et en mesurant les caractéristiques des matériaux - volume, teneur en eau à l'équilibre. Ces mesures permettent de tracer les courbes de variation d'indice des vides et de degré de saturation en fonction de la pression négative $\mathrm{u}_{w}$ (BIAREZ et al., 1988).

Pour étudier les variations de résistance en fonction de la pression, une cellule CBR à surpression d'air a été construite. Cette cellule, équipée à la base d'une membrane cellulosique semi-perméable, permet d'imposer, à l'intérieur d'un échantillon de $15 \mathrm{~cm}$ de diamètre et $15 \mathrm{~cm}$ de hauteur, une pression négative comprise entre 10 et $2500 \mathrm{kPa}$. Une fois l'équilibre atteint - généralement au bout de 1 à 2 mois - la mesure de l'indice CBR est faite dans la même cellule, dans les conditions usuelles (PARAIRE, 1987). En outre, des mesures complémentaires de pression ont été faites à l'intérieur des échantillons compactés en utilisant la technique du papier-filtre (ZERHOUNI, 1991).

Dans ce cas, il suffit de placer le papier-filtre étalonné dans l'échantillon, avant le compactage. Une fois atteint l'équilibre de pression entre le papier-filtre et l'échantillon - de l'ordre de 3 à 5 jours - la mesure de la teneur en eau de ce dernier permet de connaître, en se reportant à la courbe d'étalonnage déterminée dans les conditions de l'essai, la pression dans Yéchantillon.

\section{RÉSULTATS OBTENUS}

\subsection{Chemins d'humidification des matériaux étudiés}

Les chemins d'humidification des matériaux 2 et 3 sont représentés sur les figures 1 et 2 , où l'indice des vides est tracé en fonction de la pression négative imposée. Sur les mêmes figures ont été reportés les chemins de décompression œdométrique et les chemins de compactage à différentes teneurs en eau. On retrouve le fait que les chemins d'humidification et de décompression sont sensiblement parallèles, du moins tant que l'on ne descend pas à des valeurs de contraintes trop faibles (FLEUREAU et al., 1992). En fait, dans le cas du matériau 2, la différence importante entre les contraintes de préconsolidation, pour les courbes de décompression et d'humidification, permet d'expliquer l'écart entre les pentes des deux courbes (NASKOS, 1985). Il est donc possible, en première approximation, de déduire la relation entre la teneur en eau et la pression négative de la limite de liquidité du matériau (BIAREZ et FAVRE, 1975). On

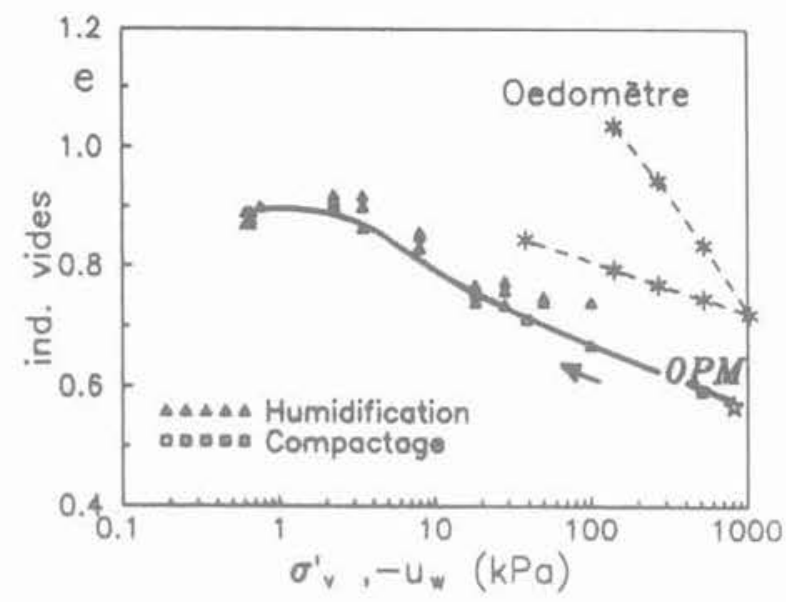

Fig. 1. - Comparaison entre les chemins d'humidification à partir de l'OPM, de décompression cedométrique et de compactage pour le matériau 2 (80\% kaolinite $+20 \%$ sable)

Fig. 1. - Comparison between the wetting path starting from the MOP, the cedometric decompression and the compaction paths for material 2 $180 \%$ kaolinite $+20 \%$ sand $)$

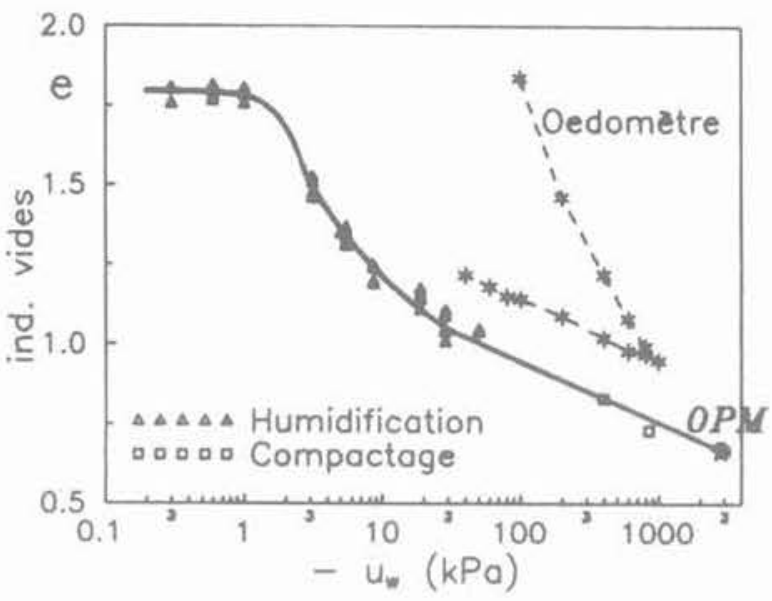

Fig. 2. - Comparaison entre les chemins d'humidification à partir de I'OPM, de décompression cedométrique et de compactage pour le matériau 3 ( $80 \%$ bentonite $+20 \%$ sable).

Fig. 2. - Comparison between the wetting path starting from the MOP, the cedometric decompression and the compaction paths for material 3 $180 \%$ bentonite $+20 \%$ sand)

remarque aussi que les points de compactage, pour les teneurs en eau supérieures à $W_{O P M}$ sont situés sur le chemin d'humidification, ce qui fournit un moyen simple d'obtenir la courbe d'humidification du sol compacté.

\subsection{Résistance CBR des matériaux}

Les figures 3 à 5 représentent les variations de différents paramètres mesurés après compactage en fonction de la teneur en eau : densité sèche, résistance $\mathrm{CBR}$ normalisée, pression négative. Dans tous les cas, la résistance CBR diminue lorsque la teneur en eau 

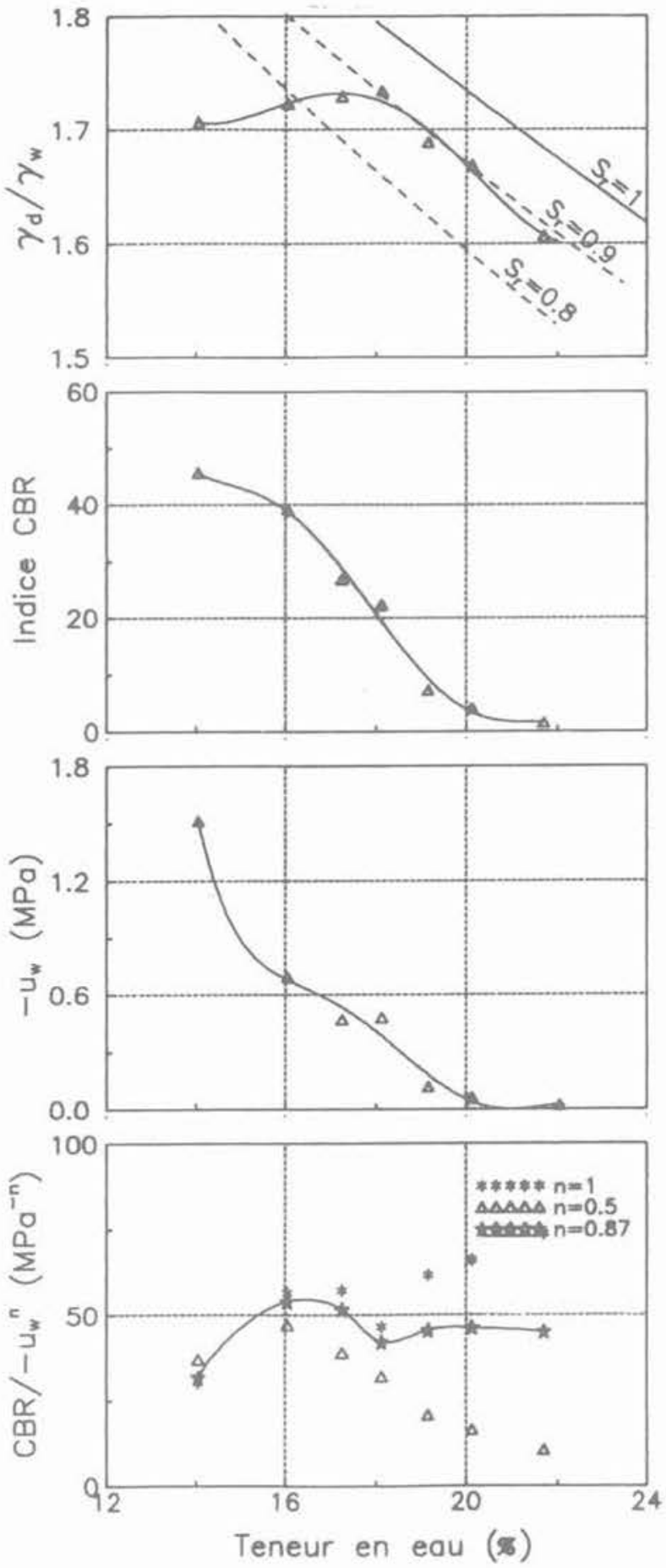

Fig. 3. - Courbes de compactage du matériau 1 : densité sèche, résistance CBR, pression négative et rapport $C B R /\left(-u_{w}\right)^{n}$ en fonction de la teneur en eau.

Fig. 3. - Compaction curves for material 1 : dry density, CBR strength, negative pressure and ration $C B R \|\left(-u_{w}\right)^{n}$ ys water content.

augmente, depuis un point situé largement (4 à 5 points) à gauche de l'optimum jusqu'à la quasisaturation. Pour les teneurs en eau plus faibles, l'effet de la densité devient prépondérant et fait chuter la résistance. La pression négative, quant à elle, présente une allure exponentielle décroissante lorsque la teneur
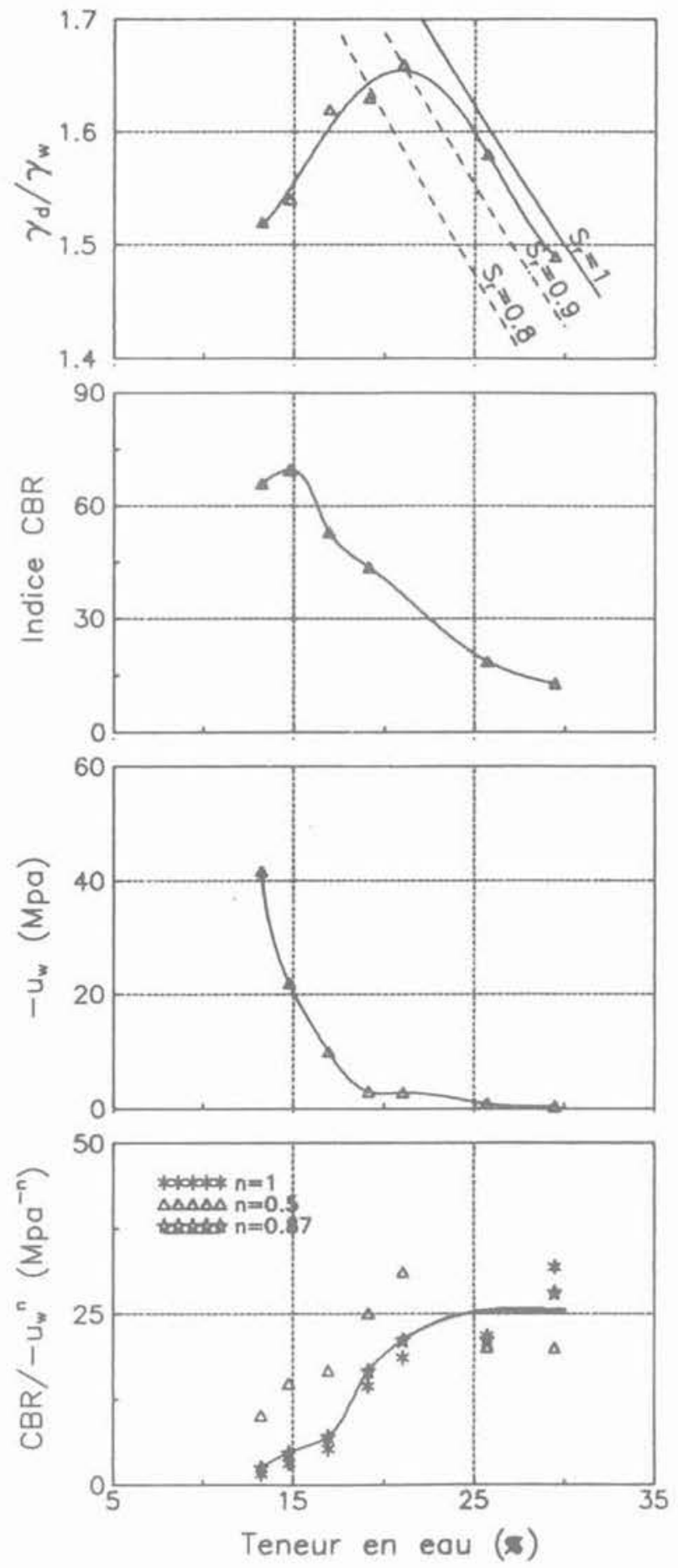

Fig. 4. - Courbes de compactage du matériau 3.

Fig. 4. - Compaction curves for material 3.

en eau augmente. Une corrélation a été recherchée entre la résistance CBR et la pression négative. La figure 6 , sur laquelle ont été reportés les points expérimentaux des trois matériaux étudiés dans la partie décroissante des courbes $\mathrm{CBR}$, montre que la résistance peut s'exprimer sous la forme :

$$
\text { CBR }=A\left(-u_{w}\right)^{n} \text {, avec } n=0,87
$$

Pour vérifier la validité de cette relation, on a tracé sur les figures 3 à 5 le rapport $C B R /\left(-u_{w}\right)^{n}$ en 

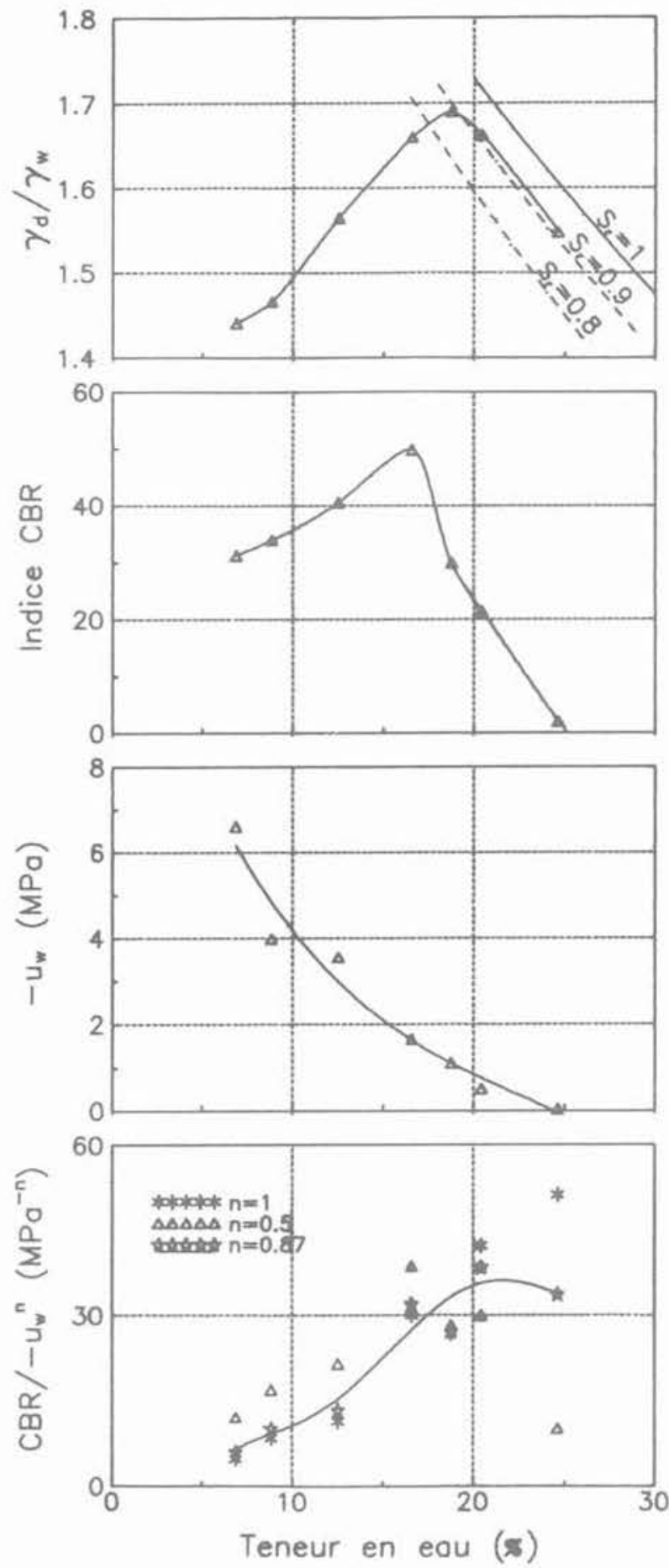

Fig. 5. - Courbes de comptage du matériau 2.

Fig. 5. - Compaction curves for material 2

fonction de la teneur en eau. On constate que, dans tous les cas, la valeur $\mathrm{n}=0,87$ conduit à un rapport sensiblement constant. Cette valeur est intermédiaire entre les valeurs caractéristiques de la plasticité parfaite $(n=1)$ et de l'élasticité linéaire $(n=0,5)$.

En effet, si l'on considère l'enfoncement du piston $\mathrm{CBR}$ comme l'enfoncement d'une fondation, la relation de TERZAGHI montre que la force portante, à une profondeur donnée, est proportionnelle à la cohé-

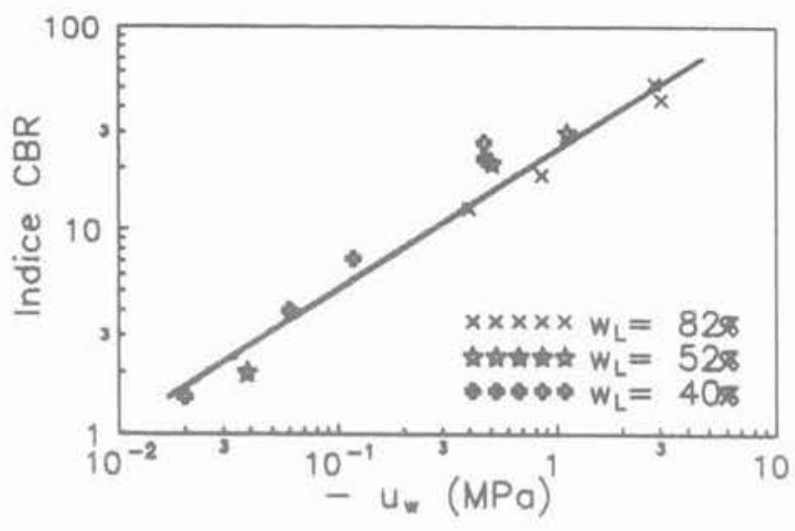

Fig. 6. - Corrélation entre la résistance CBR et la pression négative pour l'ensemble des 3 matériaux.

Fig. 6. - Correlation between the CBR strength and the negative pressure for the 3 materials.

sion apparente du sol. Pour un matériau non saturé, cette cohésion capillaire résulte de la pression interstitielle négative sous le poinçon, et l'on a :

$\mathrm{c}_{\text {cap }}=-\mathrm{u}_{\mathrm{w}} \operatorname{tg} \phi^{\prime} ;$ c'est-à-dire CBR $=A\left(-\mathrm{u}_{\mathrm{w}}\right)$

(TAIBI, 1992).

En revanche, si les déformations du sol sont élastiques linéaires, nous avons montré (BIAREZ et al., 1991) que le module d'Young était proportionnel à la racine carrée de la contrainte isotrope effective, c'est-à-dire, en première approximation, proportionnel à la racine carrée de $-\mathrm{u}_{\mathrm{w}}$ pour une contrainte totale faible. On peut donc écrire, en petites déformations :

$$
C B R=A\left(-u_{w}\right)^{0,5} .
$$

L'indice CBR apparaît donc comme la mesure d'un paramètre de la loi rhéologique du sol en moyenne déformation, dépendant de la pression interstitielle et de la contrainte intergranulaire.

\subsection{Sensibilité à l'eau des matériaux routiers}

Afin de compléter cette étude de la sensibilité à l'eau des matériaux routiers, des données complémentaires ont été rassemblées, provenant d'une part d'un chantier de route à Madagascar, d'autre part de différents chantiers de la région parisienne. Ces données comportent les mesures de caractérisation des maté riaux (granulométrie, plasticité), ainsi que les valeurs, à l'Optimum Proctor Modifié, de teneur en eau, de densité sèche, d'indice CBR immédiat et après immersion du sol pendant quatre jours. La première conclusion de cette étude a été de confirmer les résultats de différents auteurs (ROMAN ALBA, 1973: NOIRET, 1973 ; FAVRE, 1974) montrant qu'il n'était pas possible de corréler l'indice CBR après compactage d'un sol avec les autres caractéristiques à l'OPM. Comme nous l'avons montré dans les paragraphes précédents, ceci traduit simplement le fait que l'indice CBR doit être corrélé avec la pression négative plutôt qu'avec les autres paramètres d'état. En revanche, la sensibilité à l'eau peut être déduite du CBR après immersion $\left(\mathrm{CBR}_{\mathrm{sat}}\right)$. Compte tenu de l'hétérogénéité de l'échantillon étudié, qui contient à la fois des sables 
propres et des argiles plastiques, le tracé du $\mathrm{CBR}_{\text {sat }}$ en fonction de la limite de liquidité ne permet pas de classer les matériaux car les points sont très dispersés (fig. 7). Le pourcentage (F) des fins inférieurs à 80 $\mu \mathrm{m}$ apparaît comme un paramètre beaucoup plus sélectif (fig. 8), de même que l'indice de groupe $\left(I_{g}\right)^{*}$ qui tient compte à la fois de la granulométrie et de la plasticité des matériaux (fig. 9).

Les figures 8 et 9 font apparaitre l'existence de deux familles de points:

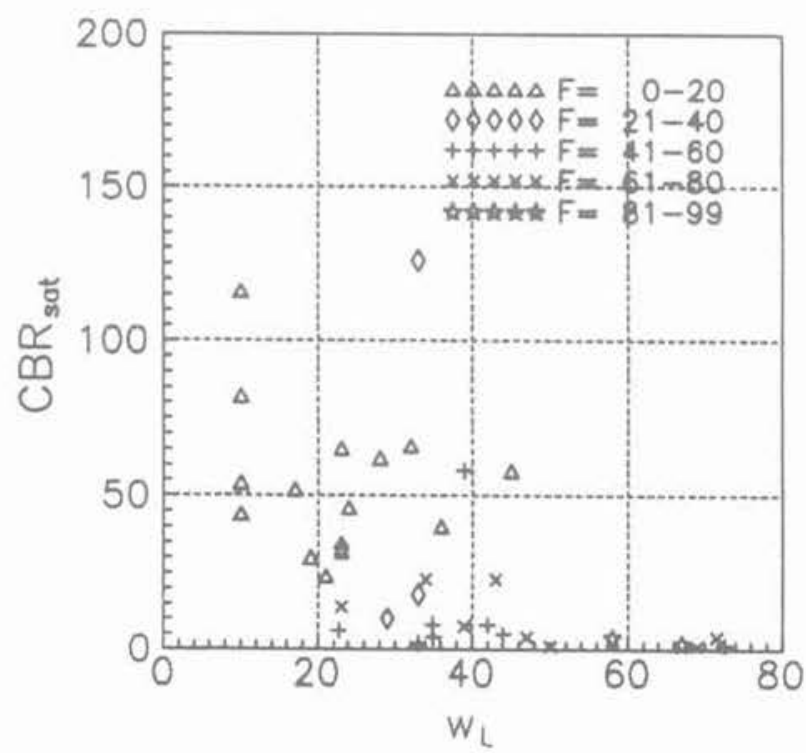

Fig. 7. - Corrélation sur un grand nombre de mesures entre la limite de liquidité et l'indice CBR après 4 jours d'immersion dans l'eau.

Fig. 7. - Correlation on a large number of data between the liquid limit and the CBR ratio after 4 days immersion in water.

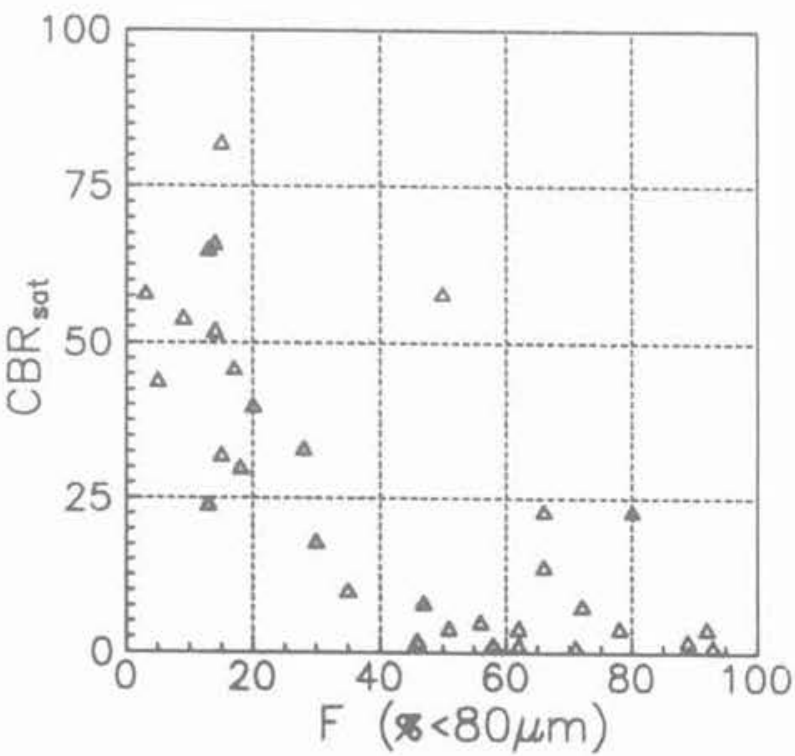

Fig. 8. - Corrélation entre le pourcentage de fins $(<80 \mu \mathrm{m})$ et le CBR après immersion.

Fig. 8. - Correlation between the percentage of fines $1<80 \mu \mathrm{m}$ and the CBR after immersion.

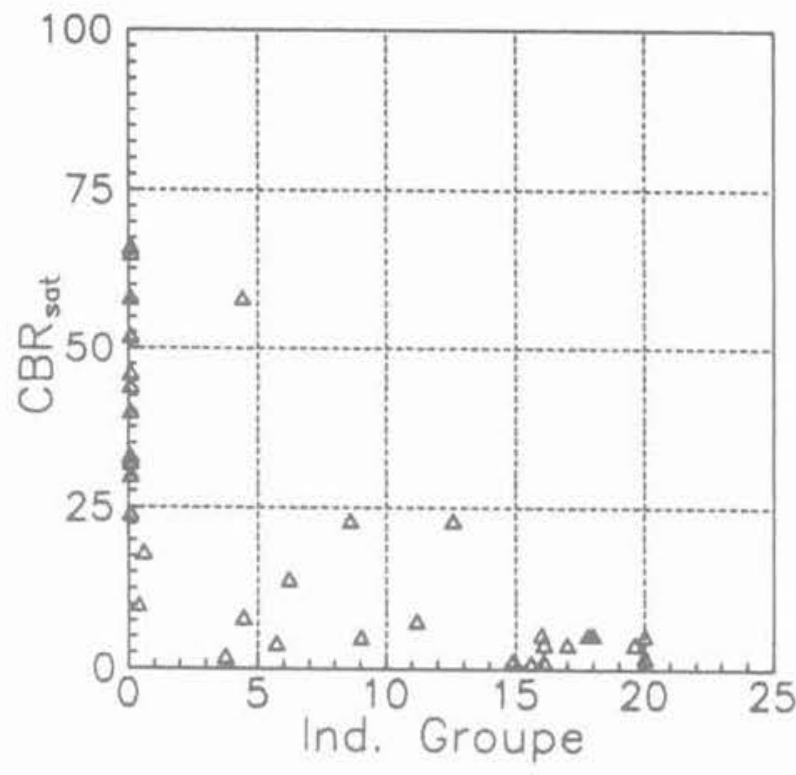

Fig. 9. - Corrélation entre l'indice de groupe et le CBR après immersion.

Fiq. 9. - Correlation between the group index and the CBR after immersion

- d'une part les matériaux dont l'indice de groupe est inférieur à 3 , quî regroupent des sols à faible pourcentage de fins $(\mathrm{F}<40 \%)$ : ces matériaux sont peu sensibles à l'eau et conservent après immersion des valeurs élevées de résistance CBR $(C B R>25)$. Pour ces matériaux, lindice de groupe est un mauvais paramètre de classement alors que le pourcentage de fins apparaît mieux adapté ;

- d'autre part les matériaux dont l'indice de groupe est supérieur à 5 ou dont le pourcentage de fins est supérieur à $40 \%$. On peut distinguer dans cette famille un premier ensemble de points $\left(5<\mathrm{I}_{\mathrm{G}}<15\right)$ pour lesquels $\mathrm{CBR}_{\mathrm{sat}}$ conserve des valeurs comprises entre 5 et 25 , et un second ensemble $\left(I_{G}>15\right)$ correspondant à des $\mathrm{CBR}_{\text {sat }}$ inférieurs à 5 . Pour ces derniers, le classement en indice de groupe devrait être remplacé par un classement en fonction des limites d'Atterberg. Bien entendu, ces derniers matériaux. très sensibles à l'eau, ne conviennent pas à la construction des chaussées.

\section{CONCLUSION}

En dépit de son caractère empirique, l'essai CBR apparaît, en raison même de sa simplicité, comme un

- L'indice de groupe est défini à partír des résultats des essais d'identification du sol, par la relation suivante :

$$
\mathrm{I}_{\mathrm{G}}=0.2 \mathrm{a}+0,005 \mathrm{a} \cdot \mathrm{c}+0,01 \mathrm{bd}
$$

dans laquelle

a. $F<35: a=0: 35<F<75$ :

$a=F-35 F:>75: a=40$

b. $F<15: b=0 ; 15<F<55$ :

$b=F-15 ; F>55: b=40$

c. $W_{L}<40: c=0 ; 40<W_{l}<60$ :

$c=W_{L}-40 ; W_{L}>60: c=20$

d. Ip $<10: \mathrm{d}=0: 10<\mathrm{lp}<30$

$\mathrm{d}=\mathrm{Ip}-10 ; \mathrm{lp}>30: \mathrm{d}=20$ 
essai mécanique bien adapté à l'étude des chaussées et de la sensibilité à l'eau des sols-supports, notamment dans les pays en voie de développement. Il permet également, en utilisant des corrélations, d'estimer approximativement la valeur du module élastique du sol à prendre en compte dans un calcul courant de vérification. Parallèlement, d'autres essais de laboratoire beaucoup plus sophistiqués (mesures en petites déformations, à la colonne résonnante, au triaxial cyclique) permettent de mesurer ce module beaucoup plus précisément, mais au prix d'une complexité parfois difficilement compatible avec l'étude d'une route. Ils apportent néanmoins des données indispensables pour la prise en compte de la non-linéarité des modules élastiques avec la contrainte ou de l'effet d'une humidification partielle de la chaussée, et ouvrent la voie vers les calculs de chaussée par éléments finis (ZERHOUNI, 1991).

Dans ce contexte, cet article apporte des éléments pour une meilleure utilisation des résultats de l'essai $\mathrm{CBR}$, mais également, au-delà de l'essai $\mathrm{CBR}$, une meilleure compréhension de l'effet de l'eau dans une chaussée ou dans une fondation de voie ferrée.

Pour les matériaux étudiés, les points de compactage à droite de l'OPM sont situés sur la courbe d'humidification du sol à partir de l'OPM, ce qui signifie que le chemin d'humidification du sol peut être approché par des essais de compactage à teneur en eau croissante. Cette courbe est sensiblement parallèle, dans le domaine des contraintes usuelles, à la courbe de décompression œdométrique et peut être estimée à partir des corrélations.

Dans ce domaine, où le concept de contrainte effective de TERZAGHI peut s'appliquer, une corrélation entre la résistance CBR et la pression négative a été établie

$$
\mathrm{CBR}=\mathrm{A}\left(-\mathrm{u}_{w}\right)^{\mathrm{n}} \text {, avec } \mathrm{n}=0,87
$$

A partir de cette relation, il est possible de prévoir l'évolution de la résistance CBR lors d'une humidification à partir des valeurs initiale et finale de pression négative.

La șensibilité à l'eau peut être étudiée à partir du CBR après immersion ; l'indice de groupe apparaît généralement comme le meilleur paramètre de classement des sols routiers à cet égard. Cependant, lorsque le pourcentage de fins est élevé, la limite de liquidité semble mieux adaptée.

Pour des sols tels que $F<15 \%$, le $C B R$ après immersion est supérieur à 25. En revanche, lorsque $\mathrm{F}>50 \%$, la probabilité d'avoir des CBR après immersion inférieurs à 5 est élevée et proscrit l'utilisation de ces matériaux pour les routes. Les matériaux intermédiaires ont des valeurs d'indice CBR après immersion qui décroissent lorsque la limite de liquidité et le pourcentage de fins augmentent.

\section{BIBLIOGRAPHIE}

BIAREZ J., FAVRE J.L. (1975), Parameters filing and statistical analysis of data in soil mechanics. Proceedings of the 2 nd Int. Conf. on Application of statistics and probabilities in soil mechanics, Aachen. vol. 2, pp. 249-264.

BIAREZ J., FLEUREAU J.M., ZERHOUNI M.I., SOEPANDJI B.S. (1988), Variation de volume des sols argileux lors de cycles de drainagehumidification. Revue Française de Géotechnique, vol. 41 , pp. 63-71.

BIAREZ J., FLEUREAU J.M., KHEIRBEK-SAOUD S. (1991), Validité de $\sigma^{\prime}=\sigma-u_{w}$ dans un sol compacté. Proceedings of the 10th European conference on soil mechanics and foundation engineering. Firenze, Mai, vol. 1, pp. 15-18.

BIAREZ J., FLEUREAU J.M., TAIBI S. (1992), Mechanical constitutive model of unsaturated soil made up by spheres, soumis pour publication dans Geomaterials.

BLIGHT G.E. (1965), A study of effective stresses for volume change, moisture equilibria and moisture changes in soils beneath covered areas. Butterworth, Australia.

ESCARIO V., SAEZ J. (1986), The shear strength of partly saturated soils, Geotechnique, vol. 36 , n० 3, pp. 453-456.

FAVRE J.L. (1974), Corrélations entre les caractéristiques de compactage et les propriétés mécaniques du sol compacté. Comptes rendus du colloque: la pratique du compactage, Expomat, Paris, 14 mai 1974, édité par J. BIAREZ \& J.M. FLEUREAU, Ecole Centrale de Paris.

FLEUREAU J.M., SOEMITRO R., TAIBI S. (1992), Behavior of an expansive clay related to suction. Proceedings of the 7th International conference on Expansive soils, Dallas, Août.

FLEUREAU J.M., INDARTO (1992), Comportement du limon de Jossigny remanié soumis à une pres. sion interstitielle négative, soumis pour publication dans la Revue Française de Géotechnique.

FREDLUNG D.G., MORGENSTERN N.R. (1977), Stress state variables for unsaturated soils. Journal of the Geotechnical Engineering Division, GT5, vol. 103, $n^{\circ} 3$, pp.261-275.

GEORGE L.A. (1988), Les effets de leau sur les chaussées, drainage et imperméabilisation. Comptes rendus du colloque : réhabilitation des réseaux routiers départementaux et communaux, Ecole Nationale des Ponts et Chaussées.

GULHATI S.K., SATIJA B.S. (1981), Shear strength of partially saturated soils. Proceedings of the 10th International conference on Soil mechanics and foundation engineering, Stockholm, vol. 1, pp. 609-612.

MATHYAS E.L., RADHAKRISHNA H.S. (1968), Volume change characteristics of partially satura. ted soils, Géotechnique, vol. 18, pp. 432-448.

NASKOS N. (1985), Quelques aspects du compor. tement mécanique de largile saturée consolidée sous fortes pressions. Thèse présentée à l'Ecole Centrale de Paris, soutenue le 18 janvier 1985 , pour obtenir le titre de Docteur-Ingénieur.

NOIRET Y. (1973), Compléments sur l'analyse en composantes principales appliquées aux sols. Bull. 
Liaison Labo. des Ponts et Chaussées, $n^{\circ} 65$, mai-juin.

PARAIRE J. (1987), Essais de succion sur des éprouvettes de diamètre $C B R$. Relation portance succion. Bull. Liaison Labo. des Ponts et Chaussées, $\mathrm{n}^{\circ} 147$, janvier-février.

ROMAN ALBA R. (1973), Proposition pour une nouvelle classification. Bull. Liaison Labo. des Ponts et Chaussées, $n^{\circ} 65$, mai-juin.

SYLVESTRE P. (1986), Drainage routier, rôle de l'environnement hydrique. Bull. Liaison Labo. des Ponts et Chaussées, $\mathrm{n}^{\circ} 145$, septembre-octobre.
TAIBI S. (1992), Comportement hydraulique et mécanique des sols non saturés. Thèse en cours à l'Ecole Centrale de Paris.

TOLL D.G. (1990), A framework for unsaturated soil behaviour. Geotechnique, vol. $40, \mathrm{n}^{\circ} 1$, pp. 35-38.

ZERHOUNI M.I. (1991), Rôle de la pression interstitielle négative dans le comportement des sols. Application au calcul des routes. Thèse présentée à l'Ecole Centrale de Paris, soutenue le 7 janvier 1991, pour obtenir le titre de Docteur. 